\title{
Los nuevos informes ampliados de auditoría. Caso: las empresas cotizadas españolas*
}

\author{
The new extended audit reports. Case: The Spanish listed companies
}

Os novos relatórios ampliados de auditoria. Estudo de caso: as empresas cotadas espanholas

\author{
Yolanda Pérez-Pérez ab \\ Universidad Complutense de Madrid, España \\ yolape01@ucm.es \\ Maria del Mar Camacho Miñano \\ Universidad Complutense de Madrid, España \\ ORCID: http://orcid.org/0000-0001-5852-338X \\ Maria Jesús Segovia Vargas \\ Universidad Complutense de Madrid, España \\ ORCID: http://orcid.org/0000-0002-6578-8017
}

DOI: https://doi.org/10.11144/Javeriana.cc20-49.ania

Fecha de recepción: 05 Noviembre 2018

Fecha de aprobación: 27 Mayo 2019

Fecha de publicación: 30 Junio 2019

\section{Resumen:}

La mayor complejidad del entorno regulatorio y de negocio ha llevado a los grupos de interés a exigir mayor confianza sobre la veracidad de la información financiera al auditor. En este contexto, el Consejo Internacional de Normas de Auditoría y Aseguramiento (IAASB) aprobó una nueva norma internacional que modificaba la estructura y los contenidos de los informes de auditoría. Uno de los principales cambios ha sido la obligación del auditor de describir en su informe las cuestiones clave de auditoría (KAM), relacionadas con riesgos significativos de la información financiera. El objetivo de este estudio es analizar el contenido del informe en el primer año de aplicación en España para 131 empresas cotizadas. Estas firmas reportan entre dos y cuatro KAM, destacando el reconocimiento de ingresos, el deterioro del fondo de comercio y la recuperación de impuestos diferidos. Este estudio tiene importantes implicaciones en otros países que van a afrontar esta reforma.

Códigos JEL: G33, M42

Palabras clave: informe de auditoría, gestión continuada, salvedades.

\section{Abstract:}

The greater complexity of the regulatory and business environment has led stakeholders to demand greater assurance on financial information from the auditor. In this context, the International Auditing and Assurance Standards Board (IAASB) adopted a new international standard on audit reports. One of the main changes has been the auditor's obligation to describe in his report the key audit matters (KAM) related to significant financial reporting risks. The objective of this study is to analyse the content of the report in the first year of application of the standard in Spain for 131 listed companies. These firms report between two and four KAM, highlighting revenue recognition, impairment of goodwill and recovery of deferred taxes. Our study has important implications in other countries that will face this reform as the United States, and can serve as a comparison to what happens in other countries.

JEL Codes: G33, M42

Keywords: audit report, going concern, qualification.

\section{Resumo:}

A maior complexidade do entorno regulatório e de negócios tem levado aos grupos de interesse a exigir maior confiança sobre a veracidade da informação financeira dada ao auditor. Neste contexto, o International Auditing and Assurance Standards Board (IAASB) aprovou uma nova norma internacional que modifica a estrutura e os conteúdos dos informes de auditoria. Uma das principais mudanças tem sido a obrigação do auditor de descrever em seu relatório as questóes relevantes de auditoria (KAM), relacionadas com riscos significativos da informação financeira. O objetivo deste estudo é analisar o conteúdo do relatório do primeiro ano de aplicação da norma em Espanha para 131 empresas cotadas. Estas firmas reportam entre dois e quatro KAM,

Notas de Autor:

\footnotetext{
Autora de correspondencia. Correo electrónico: yolape01@ucm.es

b http://www.linkedin.com/in/yolanda-perez-23b12414
} 
com destaque ao reconhecimento de ingressos, a deterioração do fundo de comércio e a recuperação de impostos diferidos. Este estudo pode ter importantes implicações em outros países que irão assumir esta reforma.

Códigos JEL: G33, M42

Palavras-chave: informe de auditoría, gestión continuada, salvedades, salvedades.

\section{INTRODUCCIÓN}

Tras la crisis financiera y la creciente complejidad en la información financiera, los inversores y otros usuarios de dicha información han demandado un informe de auditoría que proporcione información más relevante. En este contexto, el Consejo de Normas Internacionales de Auditoría y Aseguramiento (IAASB), consciente de la necesidad de cambiar el enfoque de los informes de auditoría, aprobó una norma internacional que incorporaba cambios importantes en la información contenida en estos informes (IFAC, 2013). Uno de los principales cambios ha sido la obligación de las empresas cotizadas de describir las cuestiones clave de auditoría (KAM) y el trabajo de auditoría realizado en relación con dichos asuntos (KPMG, 2016). Estas KAM son riesgos de incorrecciones materiales identificados por el auditor y que tienen un mayor efecto en la estrategia de auditoría, los recursos necesarios y el trabajo del equipo de auditoría, pero que no necesariamente conllevan a una opinión desfavorable.

Este estudio analiza de forma práctica y sistemática el contenido del informe ampliado de auditoría con especial foco en las KAM descritas en el primer año de aplicación de la norma en España para las 131 empresas cotizadas. Para ello, se elaboró una base de datos a partir de los informes de auditoría publicados a través de la comisión que controla los mercados de valores en España [http://www.cnmv.es] y la clasifi cación de las KAM realizada por el Instituto de Censores Jurados de Cuentas de España [www.icjce.es], agrupación de auditores y censores al estilo del Instituto Mexicano de Contadores Públicos -IMCP-. Desde esta perspectiva se realizó un análisis del contenido del informe de auditoría en tres bloques basados en: (i) párrafo de opinión, empresa en funcionamiento y párrafo de énfasis; (ii) KAM asociadas con los estados financieros y (iii) KAM asociadas a riesgos contables generales.

Este es un estudio pionero en analizar las KAM de las compañías cotizadas en España después de la Reforma de Auditoría que entró en vigor para ejercicios que cierren a partir del 15 de diciembre de 2016. Los primeros informes de auditoría ampliados fueron públicos a lo largo del primer y segundo trimestre de 2018. La investigación previa sobre el impacto de los informes de auditoría ampliados se ha llevado a cabo principalmente en países anglosajones.

Se espera que este trabajo motive la discusión de mecanismos adicionales para mejorar el contenido del informe en España y en otras jurisdicciones. Además, los reguladores, analistas, auditores, gerentes e inversores podrían beneficiarse de los resultados de este estudio, que presenta un análisis de los riesgos en la informaciónfinanciera a través del informe de auditoría después de las reformas del IAASB (2015) y, por lo tanto, información clave para tomar mejores decisiones empresariales y mejorar la relevancia de la auditoría.

El artículo se estructura de la siguiente manera: en primer lugar, se exponen los cambios más relevantes en el informe de auditoría tras la reforma en términos generales y en particular en los países que fueron pioneros en su implementación en Europa como son Reino Unido y Holanda, así como el caso de España, que es el país objeto de estudio. A continuación, se plantea la investigación, con el desarrollo de la muestra, las variables del estudio y la metodología empleada. Tras los resultados y su discusión, se exponen las principales conclusiones. 


\section{EL NUEVO MARCO REGULATORIO Y REVISIÓN DE LA LITERATURA}

El informe del auditor es el documento clave para comunicar los resultados del proceso de auditoría a los grupos de interés. La tabla 1 resume las responsabilidades, necesidades y valor del informe de auditoría de los grupos de interés.

TABLA 1

Relación entre los grupos de interés y el informe de auditoria

\begin{tabular}{|c|c|c|c|}
\hline $\begin{array}{l}\text { Grupos de } \\
\text { interés }\end{array}$ & Responsabilidad & Necesidades & $\begin{array}{c}\text { Valor del informe de } \\
\text { auditoría }\end{array}$ \\
\hline $\begin{array}{l}\text { a) Órganos de } \\
\text { gobierno }\end{array}$ & $\begin{array}{l}\text { Supervisar el } \\
\text { desempeño de la } \\
\text { empresa. } \\
\text { Proporcionar } \\
\text { información a los } \\
\text { grupos de interés } \\
\text { sobre el riesgo de } \\
\text { la información } \\
\text { financiera. }\end{array}$ & $\begin{array}{l}\text { Buscar la } \\
\text { información } \\
\text { especifica de la } \\
\text { empresa y la } \\
\text { transparencia. }\end{array}$ & $\begin{array}{l}\text { Fiabilidad de la } \\
\text { información } \\
\text { relacionada con la } \\
\text { consecución de los } \\
\text { objetivos de } \\
\text { negocio. }\end{array}$ \\
\hline $\begin{array}{l}\text { b) Alta } \\
\text { dirección }\end{array}$ & $\begin{array}{l}\text { Proporcionar } \\
\text { información } \\
\text { sobre las } \\
\text { discusiones } \\
\text { Significativas con } \\
\text { la gerencia en } \\
\text { relación con el } \\
\text { riesgo y las KAM. }\end{array}$ & $\begin{array}{l}\text { Buscar una } \\
\text { regulación más } \\
\text { clara con respecto a } \\
\text { las KAM. }\end{array}$ & $\begin{array}{l}\text { Fiabilidad de la } \\
\text { información } \\
\text { relacionada con el } \\
\text { logro de los } \\
\text { objetivos de la } \\
\text { presentación de } \\
\text { informes. }\end{array}$ \\
\hline Auditores & $\begin{array}{l}\text { Auditar la firma. } \\
\text { Evaluar y } \\
\text { comunicar si los } \\
\text { estados } \\
\text { financieros } \\
\text { representan su } \\
\text { imagen fiel. } \\
\end{array}$ & $\begin{array}{l}\text { Exigir una } \\
\text { regulación y } \\
\text { directrices más } \\
\text { específicas } \\
\text { relacionadas con su } \\
\text { trabajo. }\end{array}$ & $\begin{array}{l}\text { Aumento de su } \\
\text { reputación y } \\
\text { credibilidad en el } \\
\text { mercado. }\end{array}$ \\
\hline $\begin{array}{l}\text { d) } \\
\text { Reguladores }\end{array}$ & $\begin{array}{l}\text { Establecer el } \\
\text { marco de } \\
\text { información } \\
\text { financiera y la } \\
\text { Supervisión de } \\
\text { los auditores. }\end{array}$ & $\begin{array}{l}\text { Supervisar mejor } \\
\text { los intereses de los } \\
\text { grupos de interés } \\
\text { para aumentar la } \\
\text { confianza en la } \\
\text { información } \\
\text { financiera y en la } \\
\text { auditoria. }\end{array}$ & $\begin{array}{l}\text { Mayor } \\
\text { transparencia y } \\
\text { claridad en la } \\
\text { aplicación de las } \\
\text { normas de } \\
\text { contabilidad y } \\
\text { auditoria. }\end{array}$ \\
\hline $\begin{array}{l}\text { e) } \\
\text { Accionistas y } \\
\text { Otros grupos de } \\
\text { interés }\end{array}$ & $\begin{array}{l}\text { Socios de } \\
\text { negocios de la } \\
\text { Firma. }\end{array}$ & $\begin{array}{l}\text { Exigir más } \\
\text { información } \\
\text { relevante de las } \\
\text { empresas y sus } \\
\text { auditores. }\end{array}$ & $\begin{array}{l}\text { Asegurar la } \\
\text { información sobre } \\
\text { los riesgos de las } \\
\text { inversiones y los } \\
\text { préstamos. }\end{array}$ \\
\hline
\end{tabular}

Fuente: elaboración propia.

Durante muchos años se ha cuestionado el valor del informe de los auditores externos (Asare \& Wright, 2012; Carson et al., 2013). Esta preocupación se debe a que, a pesar del trabajo de los auditores, el usuario de dicho informe no lo percibe como una fuente fiable de información, ya que han surgido muchos escándalos contables en los mercados de capitales en los que empresas con informes de auditoría sin salvedades han quebrado por fraudes contables (Sikka, 2009). Históricamente, el informe de auditoría se ha descrito como un modelo de aprobado/suspenso porque ambas opiniones contienen una redacción muy estandarizada. La 
mayoría de los reguladores y bolsas de valores exigen que las empresas reciban opiniones favorables, lo que hace que casi todas las empresas que cotizan en bolsa reciban esencialmente el mismo informe de auditoría favorable (Butler et al., 2004; ICAEW, 2007; Mock et al., 2009; Coram et al., 2011; Gray et al., 2011; Czerney et al., 2014; Lennox, 2015). Los inversores han dicho que estos informes de auditoría estandarizados transmiten poca información específica de la compañía y que los auditores poseen información no pública que podría serles útil si la revelasen (PCAOB, 2013; PCAOB, 2017).

Desde 2013, el IASSB, emisor de los estándares internacionales de auditoría, ha llevado a cabo una reforma de las normas de auditoría de los informes con un nuevo enfoque, dirigido a mejorar y aumentar la información contenida en el informe de auditoría. Como consecuencia de este proceso de reforma que ha culminado con la emisión y publicación de diversas normas -entre las más relevantes, la nueva Norma Internacional de Auditoría (NIA) 701 Comunicación de los aspectos clave de la auditoría en el informe emitido por un auditor independiente-, los auditores que realicen auditorías de acuerdo con la NIA, se ven obligados a describir en el informe de auditoría de las sociedades cotizadas las KAM y el trabajo de auditoría realizado en relación con ellas. En concreto, esta NIA trata de la responsabilidad que tiene el auditor de comunicar las KAM en el informe de auditoría. Su finalidad es la de tratar tanto el juicio del auditor en relación con lo que se debe comunicar en el informe de auditoría como la estructura y contenido de dicha comunicación. En la tabla 2 se presenta una comparación de la estructura del nuevo informe de auditoría y el informe previo a la reforma.

TABLA 2

Comparación de la estructura del nuevo informe de auditoría y el informe previo a la reforma

\begin{tabular}{ll}
\hline Informe previo a la reforma & $\begin{array}{l}\text { Nuevo informe de auditoría bajo NIA } \\
\text { 701 }\end{array}$ \\
\hline Responsabilidad de la gerencia & Opinión \\
\hline Responsabilidad del auditor & Bases de opinión \\
\hline Bases de opinión & Empresa en funcionamiento \\
\hline Opinión & Cuestiones clave de auditoria (KAM) \\
\hline & Otra información \\
\hline & Responsabilidad de la gerencia \\
\hline & Responsabilidad del auditor \\
\hline
\end{tabular}

Fuente: elaboración propia

En la tabla 3 se presenta el contenido del nuevo informe de auditoría bajo la NIA 701, dado que es el objeto de este estudio. 
TABLA 3

Contenido del nuevo informe de auditoria

\begin{tabular}{|c|c|}
\hline Sección & Descripción \\
\hline Opinion & $\begin{array}{l}\text { Se mantiene el contenido y se transfiere el texto desde el } \\
\text { final hasta el principio del informe. }\end{array}$ \\
\hline Bases de opinión & Incluye una declaración sobre la independencia del auditor \\
\hline Empresa en funcionamiento & $\begin{array}{l}\text { Incertidumbre material relacionada con la capacidad de la } \\
\text { entidad para continuar como empresa en funcionamiento, } \\
\text { descrita en una sección separada. }\end{array}$ \\
\hline $\begin{array}{l}\text { Cuestiones clave de auditoría (KAM) } \\
\text { - Áreas de mayor riesgo de contenerun } \\
\text { error material o riesgo significativo. } \\
\text { - Juicios relevantes del auditor en } \\
\text { relación con áreas de los estados } \\
\text { financieros que implicaron un juicio } \\
\text { significativo de la gerencia, incluidas } \\
\text { las estimaciones contables. } \\
\text { - El efecto en la auditoría de hechos o } \\
\text { transacciones significativos que han } \\
\text { tenido lugar durante el período. }\end{array}$ & $\begin{array}{l}\text { Nuevo apartado parala auditoría de las sociedades } \\
\text { cotizadas. La divulgación es opcional para las demás } \\
\text { empresas. Se requiere la siguiente información: } \\
\text { - Referencia a la nota de los estados financieros si la } \\
\text { hubiere } \\
\text { - Por qué el asunto es una KAM; } \\
\text { - Cómo se abordó la KAM en la auditoría }\end{array}$ \\
\hline Otra información & $\begin{array}{l}\text { La nueva sección que detalla la responsabilidad del auditor } \\
\text { respecto a otra información que a compaña a los estados } \\
\text { financieros y el resulta do de los procedimientos aplicados a } \\
\text { los mismos. }\end{array}$ \\
\hline Responsabilidad de la gerencia & $\begin{array}{l}\text { Se amplió para incluir una explicación de las } \\
\text { responsabilidades de la gerencia con respecto a la empresa } \\
\text { en funcionamiento }\end{array}$ \\
\hline Responsabilidad del auditor & $\begin{array}{l}\text { Una descripción más completa de las responsabilidades del } \\
\text { auditor, incluso con respecto a la empresa en } \\
\text { funcionamiento }\end{array}$ \\
\hline
\end{tabular}

Fuente: elaboración propia basada en la ISA 701.

La fecha de aplicación de la nueva normativa en España fue para los ejercicios que se iniciaron a partir del 17 de junio de 2016. En general, la mayor parte de los países de la Unión Europea han aplicado los nuevos requisitos en los ejercicios fiscales cerrados en 2017. Otros países, como el Reino Unido u Holanda, han anticipado la transición y la respuesta de los grupos de interés ha sido positiva (KPMG, 2016). El Consejo de Información Financiera (FRC) del Reino Unido introdujo cambios significativos en los requisitos de información de las empresas y de los auditores en 2013 con el fin de proporcionar a los usuarios de los estados financieros una información más transparente. Dado que el Reino Unido fue el primer país en introducir el informe de auditoría ampliado, su experiencia podría ser útil para las empresas y los auditores de otros países. Además, los requisitos del Reino Unido iban más allá de la descripción de las KAM y exigían que el auditor informara acerca de la aplicación del concepto de materialidad y el alcance de la auditoría, incluida la forma en que ha respondido a los riesgos de incorrecciones significativas y a la aplicación de la importancia relativa.

En Holanda, el Netherlands Institute for Chartered Accountants ha fomentado la aplicación voluntaria de los nuevos informes de auditoría antes de la fecha obligatoria. El entorno político neerlandés y los representantes de los inversores también han presionado fuertemente a favor de ello. Aunque una gran parte de las firmas auditoras han llevado a cabo este ejercicio cubriendo casi la mitad de entidades cotizadas en los principales índices de cotización holandeses, el Amsterdam Exchange Index (AEX) y el Amsterdam Midkap Index (AMX), los informes de auditoría ampliados se han considerado más estandarizados que en el Reino Unido, especialmente en ciertos sectores. Por lo tanto, ha habido una respuesta "muy limitada" del público en general y de los inversores al nuevo estilo de presentación de informes. 
En España, como primer paso en el proceso de adopción de la normativa europea, en julio de 2015 se publicó la Ley 22/2015 de Auditoría de Cuentas. Esta nueva Ley de auditoría preveía cambios profundos en los informes de auditoría con respecto a los requisitos actuales, en consonancia con el nuevo enfoque de la NIA. La nueva ley de auditoría ya es aplicable para los ejercicios que comenzasen a partir del 17 de junio de 2016. Como consecuencia de esta nueva normativa, el auditor tiene un nuevo papel consistente en evaluar el riesgo en la información financiera.

En Colombia, la NIA 701, introducida a las Normas de Aseguramiento de la Información a través del Decreto 2170 del 20 de diciembre de 2017, ha mostrado los lineamientos que debe tener en cuenta un contador al determinar y comunicar cuestiones clave identificadas en la labor de auditoría. En la tabla 4 se sintetizan las principales conclusiones del análisis de Debilidades, Amenazas, Fortalezas y Oportunidades (DAFO) de las experiencia revisadas de los países pioneros.

TABLA 4

DAFO del informe de auditoría ampliado

\begin{tabular}{|c|c|}
\hline Fortalezas & Debilidades \\
\hline $\begin{array}{l}\text { - Mejora de la comunicación entre los auditores y los } \\
\text { inversores, así como conlos encargados del } \\
\text { gobiemo corporativo. } \\
\text { - Mayor confianza de los usuarios en los informes de } \\
\text { auditoría y los estados financieros. } \\
\text { - Aumento de la transparencia, la calidad de las } \\
\text { auditorías y mejora del valor de la información. } \\
\text { - Mayor atención por parte de la gerencia y de los } \\
\text { preparadores de los estados fínancieros a los } \\
\text { desgloses que hacen referencia al informe del } \\
\text { auditor. } \\
\text { - Mejora de la información financiera en aras del } \\
\text { interés público. }\end{array}$ & $\begin{array}{l}\text { - No revelación de información sobre el uso } \\
\text { de la materialidad para la ejecución. } \\
\text { - No se incluyen las conclusiones del auditor } \\
\text { sobre los riesgos de incorrecciones } \\
\text { significativas. } \\
\text { - Falta de un análisis dinámico de los cambios } \\
\text { a lo largo del tiempo en cuanto a su enfoque } \\
\text { de auditoría, el nivel de importancia relativa } \\
\text { o los riesgos evaluados comunicados. }\end{array}$ \\
\hline Oportunidades & Amenazas \\
\hline $\begin{array}{l}\text { - Comentarios limitados sobre la materialidad. } \\
\text { - Mayor alineación entre los informes del Comité de } \\
\text { Auditoría y los informes de los auditores. } \\
\text { - Evolución del lenguaje utiliza do en los informes de } \\
\text { auditoría: de descripciones genénicas a unas } \\
\text { descripciones de riesgos más detalladas. } \\
\text { - Más información sobre los rangos de sensibilidad } \\
\text { utilizados en las pruebas; la evaluación del auditor } \\
\text { de la calidad de los controles intemos de una } \\
\text { entidad sobre sus riesgos signifícativos; y la } \\
\text { opinión del auditor sobre la idoneidad de las } \\
\text { estimaciones de la Dirección. } \\
\text { - Mayor transparencia sobre los supuestos de la } \\
\text { dirección y los puntos de referencia utilizados por } \\
\text { los auditores. }\end{array}$ & $\begin{array}{l}\text { - Mayores costes, particulamente para } \\
\text { aquellas entidades cuyo tamaño no es muy } \\
\text { grande. Hacer que las consideraciones de } \\
\text { coste/beneficio sean más importantes. } \\
\text { - Riesgo de aumentar la homogeneidad a lo } \\
\text { largo del tiempo, lo que da lugar a informes } \\
\text { de auditoría estándar. }\end{array}$ \\
\hline
\end{tabular}

Fuente: elaboración propia.

Luego de la revisión de la literatura sobre la auditoría se comprobó que los artículos de investigación se han centrado, fundamentalmente, en (i) el análisis del impacto de las reformas de auditoría en función de las motivaciones de los grupos de interés (Guiral-Contreras et al., 2007; Coram et al., 2011; Deumes et al., 2012; Reid et al., 2018); (ii) la calidad de la auditoría e información financiera (Kilgore et al., 2014, Garza Sánchez et al., 2016; Prasad \& Chand, 2017); (iii) el contenido, estructura y lenguaje del informe del auditor (Christensen et al., 2012; Cox, 2013; Hategan et al., 2015; Fakhfakh, 2016a) y (iv) el impacto de las KAM, para los que hay controversia entre los investigadores (Boolaky \& Quick, 2016; Brasel et al., 2016; Gutierrez 
et al., 2017; Sirois et al., 2017; Kachelmeier et al., 2019; Clive et al., 2019). Sin embargo, no se identificó ningún estudio de investigación que haya realizado el análisis del nuevo informe de auditoría ampliado en el contexto español. Este es el propósito de este artículo.

Con este estudio empírico se trata de responder a las siguientes preguntas de investigación: (i) ¿Qué tipo de contenido aporta el informe de auditoría ampliado en el primer año de implementación en las empresas cotizadas españolas? y (ii) ¿qué número y tipo de KAM son descritos en los informes de auditoría de las empresas cotizadas españolas, considerando el mercado, el tamaño de auditor y el sector auditado?

\section{ANÁLISIS DEL CONTENIDO DEL INFORME DE AUDITORÍA AMPLIADO}

\section{La muestra del estudio}

La muestra está formada por todas las empresas que cotizan en la principal Bolsa de Valores de España a 31 de diciembre de 2017 partiendo de la base de datos Orbis [http://www.informa.es/soluciones-financieras/sabi/ orbis], en total, 131. Dentro de la Bolsa de Valores en España se denomina "mercado continuo" al sistema por el que las cuatro bolsas de valores españolas se encuentran interconectadas como un único mercado bursátil (Madrid, Bilbao, Barcelona y Valencia). A él pertenecen la mayor parte de las grandes empresas españolas, siendo las 35 de mayor liquidez las que pertenecen a un índice bursátil de referencia denominado con el IBEX-35 (Iberia Index de las 35 empresas españolas con mayor liquidez). Teniendo en cuenta el total de esas 131 empresas, la cobertura por tipo de mercado es de 35 empresas (27\%) del IBEX 35 y 96 empresas (73\%) del mercado continuo.

\section{Variables del estudio}

Las variables de este estudio se han obtenido del análisis del informe ampliado y la categorización en tres bloques (A, B y C). Para el Bloque A se han identificado los distintos tipos de párrafos de opinión atendiendo a la NIA 701. Para los Bloques B y C se han identificado las KAM y se han clasificado según su naturaleza, considerando los criterios establecidos por el Instituto de Censores Jurados de Cuentas de España (ICJCE), que es una de las principales agrupaciones de profesionales y sociedades de auditoría de cuentas que operan en España. Las KAM se clasificaron en dos categorías: KAM contables, que son los relacionados con un epígrafe específico de las cuentas anuales, y KAM generales, que están relacionados con temas condicionados por factores externos.

Bloque A: Tipo de párrafo. Se han identificado tres variables en función del tipo de párrafo de opinión de auditoría: "opinión de auditoría" (OPIAU), pudiendo ser favorable o con salvedades; "énfasis" (EMPA) si refleja una cuestión presentada o revelada de forma adecuada en los estados financieros y que, a juicio del auditor, es de tal importancia que resulta fundamental para que los usuarios comprendan los estados financieros; y "Empresa en funcionamiento" (GCPA) si describe una incertidumbre material relacionada con la capacidad de la entidad para continuar como empresa en funcionamiento.

Bloque B: KAM Contables. Se han identificado 17 variables correspondientes a KAM asociadas a cada uno de los epígrafes de los estados financieros: Inmovilizado intangible (ININ), Fondo de Comercio (FC), Inmovilizado material e inversiones inmobiliarias (IMIM), Inversiones en empresas del grupo y asociadas (IEGA), Inversiones financieras (IF), Activos por impuesto diferido (AID), Existencias (INV), Otros activos corrientes (OAC), Provisiones y pasivos contingentes (PPC), Impuestos (IMP), Deudas con entidades de crédito (BAB), Otros pasivos financieros (OPF), Pensiones (PEN), Periodificaciones (PER), 
Reconocimiento de ingresos (REI), Operaciones con partes vinculadas (OPV), y Combinaciones de negocios $(\mathrm{CN})$.

Bloque C: KAM General. Se han identificado 6 variables correspondientes a KAM no asociadas a ningún epígrafe de los estados financieros concreto, sino que representan riesgos generales que afectan de manera global a los estados financieros: Sistemas de información (SI), Cumplimiento legal y normativo (CLN), Cumplimiento condiciones contractuales (CCC), Transacciones inusuales o significativas (TIS), Empresa en funcionamiento (EMP) y Otros (OTR).

\section{Metodología}

Se realizó un estudio descriptivo del contenido del informe de auditoría ampliado, que consistió en la lectura, análisis y clasificación del contenido del informe de auditoría ampliado de las 131 sociedades cotizadas existentes en el mercado español. Para cada firma se tomaron sus estados financieros, incluyendo el informe de auditoría ampliado del regulador del mercado de valores, de la página web de la Comisión Nacional del Mercado de Valores (CNMV), correspondiente al ejercicio social cerrado a 31 de diciembre de 2017. A partir de la lectura y análisis de este informe ampliado de cada una de las empresas de la muestra se desarrolló una codificación del contenido de dicho informe, una clasificación basada en el estudio de Muñoz-Izquierdo et al. (2017) que distribuye las variables del análisis en los tres bloques mencionados anteriormente. Se entiende por contenido los mensajes incluidos por el auditor tanto en la opinión, así como en los párrafos de énfasis, salvedades y KAMs.

Según los criterios mencionados del Instituto de Censores Jurados de Cuentas de España, uno de los autores codificó la información de cada uno de los informes de auditoría y, para incrementar la validez de la propuesta, otro experto hizo la misma labor, para obtener una propuesta unificada de clasificación. Para cada comentario que aparecía en los informes se creaba una categoría a la que se añadía el comentario con el valor 1 . Al resto de categorías no reflejadas en dicho informe se le asignaba valor cero, creando así variables dicotómicas. Un análisis descriptivo de dichas variables respondió la primera pregunta de la investigación: ¿qué tipo de contenido aporta el informe de auditoría ampliado en el primer año de implementación en las empresas cotizadas españolas?

Para responder la segunda pregunta, sobre qué número y tipo de KAM se describen en los informes de auditoría de las empresas cotizadas españolas, considerando el tipo de mercado, el tamaño de auditor y el sector auditado, se obtuvo información relacionada con el tipo de mercado, el tipo de auditor y el sector auditado, mediante el uso de tablas cruzadas o de doble entrada. En estas tablas se registró la relación del contenido del informe de auditoría y los tres factores que pueden condicionar dicho contenido. Para el tipo de mercado se distinguió entre IBEX-35 o mercado continuo (MC). Por el tamaño del tipo de auditor, se distinguieron cuatro categorías: 'Big4' o las cuatro grandes firmas internacionales de auditoría, que incluyen a Deloitte, EY, KPMG y PwC; 'Segundo nivel' o firmas de tamaño medio, en las que aparecen BDO Auditores, S.L. y Grant Thornton, S.L.P.; 'resto de corporaciones' u otras pequeñas firmas de auditoría en forma de sociedades mercantiles; e 'individuales', recolectadas por los auditores individuales o por las personas físicas que presten servicios de auditoría. En cuanto a la clasificación del sector, se agruparon en ocho categorías basadas en la Clasificación Nacional de Actividades Económicas -CNAE- ${ }^{1}$ : construcción e inmobiliario, consumo y distribución, energía y recursos naturales, financiero, salud, industria, servicios y tecnología, medios de comunicación y comunicaciones. 


\section{RESULTADOS Y DISCUSIÓN}

Las observaciones obtenidas se basaron en el análisis de los informes de auditoría emitidos por los auditores en España en relación con la información financiera publicada por las entidades cotizadas en las principales Bolsas de Valores españolas tras la entrada en vigor de la Reforma de Auditoría. Como parte del estudio se revisaron un total de 131 informes de auditoría, que presentaban un total de 373 KAM. A continuación se presenta el análisis realizado en relación al contenido del informe de auditoría ampliado.

\section{Frecuencia del contenido del informe de auditoría}

En la tabla 5 se detalla la frecuencia y representatividad de cada ítem de la clasificación.

TABLA 5

Frecuencia del contenido del informe de auditoria

\begin{tabular}{|c|c|c|c|c|}
\hline Variable & Explicación & Frecuencia & Bloque (\%) & Muestra (\%) \\
\hline \multicolumn{5}{|c|}{ Bloque A: Tipo de párrafo } \\
\hline OPIAU & Opinión de auditoría & 3 & $16 \%$ & $2 \%$ \\
\hline EMPA & Párrafo de énfasis & 4 & $21 \%$ & $3 \%$ \\
\hline GCPA & Párrafo de empresa en funcionamiento & 12 & $63 \%$ & $9 \%$ \\
\hline \multicolumn{5}{|c|}{ Blouse B: KAM constables } \\
\hline ININ & Inmoviliza do intangible & 13 & $3 \%$ & $10 \%$ \\
\hline $\mathrm{FC}$ & Fondo de Comercio & 50 & $13 \%$ & $38 \%$ \\
\hline IMIM & $\begin{array}{l}\text { Inmovilizado material e inversiones } \\
\text { inmobiliarias }\end{array}$ & 42 & $11 \%$ & $32 \%$ \\
\hline IEGA & $\begin{array}{l}\text { Inversiones en empresas del grupo y } \\
\text { asociadas }\end{array}$ & 4 & $1 \%$ & $3 \%$ \\
\hline IF & Inversiones financieras & 18 & $5 \%$ & $14 \%$ \\
\hline AID & Activos por impuesto diferido & 44 & $12 \%$ & $34 \%$ \\
\hline INV & Existencias & 16 & $4 \%$ & $12 \%$ \\
\hline $\mathrm{OAC}$ & Otros activos comientes & 3 & $1 \%$ & $2 \%$ \\
\hline PPC & Provisiones y pasivos contingentes & 38 & $10 \%$ & $29 \%$ \\
\hline IMP & Impuestos & 8 & $2 \%$ & $6 \%$ \\
\hline $\mathrm{BAB}$ & Deudas con entidades de crédito & 4 & $1 \%$ & $3 \%$ \\
\hline OPF & Otros pasivos financieros & 11 & $3 \%$ & $8 \%$ \\
\hline PEN & Pensiones & 3 & $1 \%$ & $2 \%$ \\
\hline PER & Periodificaciones & - & $0 \%$ & $0 \%$ \\
\hline REI & Reconocimiento de ingresos & 58 & $16 \%$ & $44 \%$ \\
\hline OPV & Operaciones con partes vinculadas & 1 & $0 \%$ & $1 \%$ \\
\hline $\mathrm{CN}$ & Combinaciones de negocios & 20 & $5 \%$ & $15 \%$ \\
\hline \multicolumn{5}{|c|}{ Bloque C: KAM General } \\
\hline SI & Sistemas de información & 11 & $3 \%$ & $8 \%$ \\
\hline CLN & Cumplimiento legal y normativo & 7 & $2 \%$ & $5 \%$ \\
\hline $\mathrm{CCC}$ & Cumplimiento condiciones contractuales & 3 & $1 \%$ & $2 \%$ \\
\hline TIS & Transacciones inusuales o significativas & 7 & $2 \%$ & $5 \%$ \\
\hline EMP & Empresa en funcionamiento & 4 & $1 \%$ & $3 \%$ \\
\hline OTR & Otros & 8 & $2 \%$ & $6 \%$ \\
\hline
\end{tabular}

Fuente: elaboración propia

En las siguientes secciones se realiza un análisis específico para mostrar el resultado de cada bloque. 


\section{Tipo de párrafo (Bloque $\mathrm{A})$}

Tipo de opinión. En el año 2017 los auditores sólo emitieron tres opiniones con salvedades. Una de ellas estaba relacionada con la empresa Dogi International Fabrics, SA, auditado por PWC, debido a las contingencias fiscales asociadas a algunas filiales y a la no constitución de provisiones registradas. Otra opinión con salvedades fue para la Compañía Levantina de Edificación y Obras Públicas, S.A., auditada por un auditor individual, debido a la incertidumbre relacionada con la quiebra de una filial significativa. Y Mobiliaria Monesa, S.A., auditada por BDO, tenía un alcance limitado sobre una filial significativa debido a la falta de información financiera relacionada con esta filial. El resto de las opiniones de los auditores eran favorables, lo que significaba que los estados financieros de las empresas cotizadas reflejaban de manera fiel su situación económica y financiera. Estas empresas han hecho un gran esfuerzo por disponer de informes favorables dado que el órgano regulador -CNMV- ha presionado para que estas empresas reflejen unos correctos estados financieros debido a su gran repercusión económica en los mercados que cotizan.

Párrafo de énfasis. Como la experiencia española de este informe ha sido para las empresas más grandes, existían pocas opiniones con salvedades así como con párrafos de énfasis. Por lo tanto, los auditores sólo identificaron cuatro párrafos de énfasis en 2017, considerando tanto el IBEX-35 como el MC. Los cuatro casos eran: Ferrovial SA (empresa constructora) en el IBEX-35 (auditada por Deloitte) para la aplicación de la CINIIF 15 "Acuerdos para la construcción anticipada de inmuebles”; Unicaja Banca SA (empresa financiera) en el MC (auditada por PWC) debido a su fusión por absorción; Amper SA (empresa de tecnología) en el MC (auditado por EY) por su acuerdo de venta del negocio de Pacífico Sur sujeto a algunas condiciones y licencias; y finalmente, Mobiliaria Monesa SA (empresa inmobiliaria) en el MC (auditado por BDO) por la incertidumbre relacionada con la quiebra de una importante subsidiaria. Sólo Mobiliaria Monesa, S.A presentó un informe con salvedades, lo cual es un indicativo de que los párrafos de énfasis sólo pretenden llamar la atención sobre aspectos relevantes contables complejos, sin que por ello suponga sistemáticamente una salvedad en el informe de auditoría.

Empresa en funcionamiento. Las normas de auditoría estipulan que los auditores deben revelar públicamente si una empresa está teniendo dificultades en su capacidad de continuar como empresa en funcionamiento ("going concern" en los países anglosajones). Estudios previos de investigación demostraron que los párrafos de empresa en funcionamiento tenían repercusiones en distintos grupos de interés. Así por ejemplo, Zenzerovic \& Valic-Vale (2016) demostraron empíricamente que las empresas con párrafos de empresa en funcionamiento tenían inferiores ratios económicos y financieros. Esto podría explicarse porque, para los actuales inversores, este párrafo proporciona al mercado información de valoración adicional específica de la empresa, además de la información ya disponible públicamente. Carson et al. (2013) demostraron que para los prestamistas este párrafo les advierte que es probable que los valores de liquidación de los activos sean muy inferiores a los valores contables en caso de quiebra.

En este estudio se observó que los auditores sólo identificaron 12 párrafos de empresa en funcionamiento en el año 2017. Tres de ellas son empresas que tenían una opinión con salvedades (ver panel A, tabla 6). Es notable que algunos auditores consideren la empresa en funcionamiento como una KAM pero no en un párrafo específico (ver panel B, tabla 6). Esta cuestión es interesante porque si se incluye este concepto de empresa en funcionamiento con el resto de las KAM, se puede ocultar el impacto de los riesgos de viabilidad de las empresas. El hecho de que la empresa en funcionamiento no se revele en los informes anuales implica una opinión con salvedades en su informe de auditoría. Si el principio de empresa en funcionamiento se revela en el informe anual, entonces los auditores deben verificarlo. Y si los auditores están de acuerdo debe revelar como una KAM, de lo contrario como un párrafo de énfasis separado para dar mayor visibilidad a los usuarios de la información financiera. 
TABLA 6

Empresa en funcionamiento en el informe ampliado del auditor externo

\begin{tabular}{|c|c|c|c|c|}
\hline \multicolumn{5}{|c|}{ Panel A: Párrafos de empresa en funcionamiento } \\
\hline Auditor & Mercado & Empresa & Sector & Empresa en funcionamiento \\
\hline PWC & MC & $\begin{array}{l}\text { Dogi International } \\
\text { Fabrics, S.A. }\end{array}$ & Servicios & $\begin{array}{l}\text { Patrimonio neto negativo; capital } \\
\text { circulante negativo }\end{array}$ \\
\hline $\begin{array}{l}\text { Luis } \\
\text { Caruana }\end{array}$ & $\mathrm{MC}$ & $\begin{array}{l}\text { Compañia Levantina } \\
\text { de Edificacióny } \\
\text { Obras Públicas, S.A. }\end{array}$ & Construcción & $\begin{array}{l}\text { Patrimonio neto negativo; capital } \\
\text { circulante negativo }\end{array}$ \\
\hline BDO & $\mathrm{MC}$ & $\begin{array}{l}\text { Mobiliaria Monesa, } \\
\text { S.A. }\end{array}$ & Inmobiliaria & $\begin{array}{l}\text { Pérdidas significativas en una } \\
\text { filial importante }\end{array}$ \\
\hline Deloitte & MC & $\begin{array}{l}\text { Obrascon Huarte } \\
\text { Lain, S.A. }\end{array}$ & $\begin{array}{l}\text { Construccióne } \\
\text { Inmobiliaria }\end{array}$ & $\begin{array}{l}\text { Financiación as ociada al } \\
\text { cumplimiento de desinversiones }\end{array}$ \\
\hline Deloitte & MC & Abengoa, S.A & $\begin{array}{l}\text { Energia y recursos } \\
\text { naturales }\end{array}$ & Patrimonio negativo \\
\hline EY & MC & Duro Felguera, S.A. & $\begin{array}{l}\text { Construccióne } \\
\text { Inmobiliaria }\end{array}$ & $\begin{array}{l}\text { Patrimonio neto negativo; capital } \\
\text { circulante negativo }\end{array}$ \\
\hline PWC & MC & Tubos Reunidos, S.A & Industrial & $\begin{array}{l}\text { Patrimonio neto negativo; capital } \\
\text { circulante negativo }\end{array}$ \\
\hline Deloitte & MC & $\begin{array}{l}\text { Corporación } \\
\text { Empresarial de } \\
\text { Materiales de } \\
\text { Construcción, S.A. }\end{array}$ & $\begin{array}{l}\text { Consumoy } \\
\text { distribución }\end{array}$ & $\begin{array}{l}\text { Desinversión en una empresa que } \\
\text { es la principal fuente de ingresos y } \\
\text { algunas contingencias }\end{array}$ \\
\hline $\begin{array}{l}\text { Baker Tilly } \\
\text { Fmac, SLP }\end{array}$ & MC & $\begin{array}{l}\text { Urbas Grupo } \\
\text { Financiero, S.A. }\end{array}$ & Servicios & $\begin{array}{l}\text { Fondo de manio bra negativo; } \\
\text { incertidumbre sobre la novación } \\
\text { de los acuerdos de financiación }\end{array}$ \\
\hline BDO & $\mathrm{MC}$ & $\begin{array}{l}\text { Urbar Ingenieros, } \\
\text { S.A. }\end{array}$ & $\begin{array}{l}\text { Construccióne } \\
\text { Inmobiliaria }\end{array}$ & $\begin{array}{l}\text { Fondo de manio bra negativo; } \\
\text { incertidumbre sobre la novación } \\
\text { de los acuerdos de financiación }\end{array}$ \\
\hline $\begin{array}{l}\text { HORWATH } \\
\text { AUDITORE } \\
\text { S ESPAN̈A } \\
\text { S.L. }\end{array}$ & MC & $\begin{array}{l}\text { Vertice Trescientos } \\
\text { Sesenta Grados, S.A. }\end{array}$ & $\begin{array}{l}\text { Tecnologia, medios } \\
\text { y } \\
\text { telecomunicaciones }\end{array}$ & Fondo de manio bra negativo \\
\hline BDO & MC & $\begin{array}{l}\text { Nyesa Valores } \\
\text { Corporacion, S.A. }\end{array}$ & Construcción & $\begin{array}{l}\text { Patrimonio por debajo delos dos } \\
\text { tercios del capital social }\end{array}$ \\
\hline \multicolumn{5}{|c|}{ Panel B: Párrafo de empresa en funcionamiento incluido como una KAM } \\
\hline Auditor & Mercado & Empresa & Sector & Empresaen funcionamiento \\
\hline Deloitte & MC & $\begin{array}{l}\text { Promotora de } \\
\text { Informaciones, S.A. }\end{array}$ & $\begin{array}{l}\text { Tecnologia, medios } \\
\text { y } \\
\text { telecomunicaciones }\end{array}$ & $\begin{array}{l}\text { Evolución de la estructura } \\
\text { patrimonial y financiera de } \\
\text { la empresa }\end{array}$ \\
\hline PWC & MC & Pharma Mar, S.A. & Servicios & Capacidad financiera \\
\hline KPMG & MC & Ecolumber, S.A. & $\begin{array}{l}\text { Energia y recursos } \\
\text { naturales }\end{array}$ & $\begin{array}{l}\text { Financiación de la actividad del } \\
\text { grupo }\end{array}$ \\
\hline BDO & $\mathrm{MC}$ & Sniace, S.A. & $\begin{array}{l}\text { Energia y recursos } \\
\text { naturales }\end{array}$ & $\begin{array}{l}\text { Aplicación del principio de } \\
\text { empresa en funcionamiento }\end{array}$ \\
\hline
\end{tabular}

Fuente: elaboración propia basada en el infome annual del auditor externo de cada compañía obtenido de la página de la CNMV.

\section{Análisis de las KAM (Bloque B y C)}

Número de riesgos. Si bien la NIAS 701 en su apartado Determinación de las cuestiones clave de la auditoría (pp. A9-A30) proporciona directrices para determinar si un asunto de auditoría constituye una KAM (ver resumen en Tabla 3), hay relativamente poca orientación con respecto al número de KAM que deben notificarse. De hecho, cuáles y cuántas KAM reportar es una cuestión de juicio profesional, de ahí la relevancia de este análisis. 


\section{- Número de riesgos por firma de auditoría}

Se recopilaron datos sobre el número de riesgos comunicados por cada firma de auditoría de esta muestra. El número total medio de riesgos reportados para las empresas del Mercado Contínuo se situó entre tres y cuatro, mientras que el Ibex-35 se situó entre dos y tres. Es notable que el mayor número de riesgos reportados por las firmas que no son Big4 sean siete, mientras que sólo tenían alrededor del 10\% del mercado de auditoría. Por lo tanto, parece que la cuota de auditoría no ha afectado al número de KAM identificados por los auditores. Sin embargo, una observación relevante al respecto es que en el caso de firmas que no son Big-4, todos los KAM descritos están asociados con epígrafes contables (KAM contables). Estos resultados están en línea con la conclusión de Sierra-Garci\#a et al. (2019) quienes demostraron que, en general, los auditores que perciben mayores honorarios (Big4) presentan mayores KAM generales y menores KAM contables en el caso británico.

\section{- Número de riesgos por sector}

También se analizó la distribución de riesgos entre los diferentes sectores, y los resultados de esta revisión se presentan en la tabla 7 .

TABLA 7

Número de riesgos por sector

\begin{tabular}{|c|c|c|c|c|c|c|c|c|}
\hline \multirow[b]{2}{*}{ Sector } & \multicolumn{2}{|c|}{ Ibex-35 } & \multicolumn{2}{|c|}{$\mathrm{MC}$} & \multicolumn{2}{|c|}{ Total } & \multicolumn{2}{|c|}{ Media } \\
\hline & $\begin{array}{l}\mathrm{N}^{\circ} \\
\text { Firm }\end{array}$ & $\begin{array}{c}\mathrm{N}^{\circ} \\
\mathrm{KAM}\end{array}$ & $\begin{array}{c}\mathrm{N}^{\circ} \\
\text { Firm }\end{array}$ & $\begin{array}{c}\mathrm{N}^{\circ} \\
\mathrm{KAM}\end{array}$ & $\begin{array}{c}\mathrm{N}^{\circ} \\
\text { Firm }\end{array}$ & $\begin{array}{c}\mathrm{N}^{\circ} \\
\mathrm{KAM}\end{array}$ & Ibex-35 & $\mathrm{MC}$ \\
\hline $\begin{array}{l}\text { Construccióne } \\
\text { Inmobiliaria }\end{array}$ & 5 & 17 & 22 & 52 & 27 & 69 & 3.4 & 2.4 \\
\hline $\begin{array}{l}\text { Consumo y } \\
\text { distribución }\end{array}$ & 2 & 5 & 6 & 12 & 8 & 17 & 2.5 & 2.0 \\
\hline $\begin{array}{l}\text { Energía y recursos } \\
\text { naturales }\end{array}$ & 8 & 32 & 8 & 22 & 16 & 54 & 4.0 & 2.8 \\
\hline Financiero & 7 & 37 & 7 & 24 & 14 & 61 & 5.3 & 3.4 \\
\hline Farmacéutica & - & & 2 & 4 & 2 & 4 & & 2.0 \\
\hline Industrial & 4 & 12 & 26 & 67 & 30 & 79 & 3.0 & 2.6 \\
\hline Servicios & 5 & 14 & 18 & 41 & 23 & 55 & 2.8 & 2.3 \\
\hline $\begin{array}{l}\text { Tecnologia, medios y } \\
\text { telecomunicaciones }\end{array}$ & 4 & 12 & 7 & 22 & 11 & 34 & 3.0 & 3.1 \\
\hline Total & 35 & 129 & 96 & 244 & 131 & 373 & & \\
\hline
\end{tabular}


Se observó que hay diferencias entre sectores. Esta conclusión está en línea con los estudios previos de Ruiz-Barbadillo et al. (2002) en España y Sierra-García, et al. (2019) para las empresas británicas que mostraban que las diferencias por sector eran debidas al riesgo inherente a cada uno de ellos que, por tanto, genera distintas complejidades contables asociadas a ellos. Por ejemplo, sectores intensivos en capital como construcción o energía, concentraban mayor portfolio de riesgos en activos fijos o contratos relacionados con cumplimiento de acuerdos. El sector financiero presentó un mayor número medio de riesgos, probablemente por los mayores requisitos de la nueva regulación financiera así como las que entrarán en vigor en los próximos años. De acuerdo con un estudio de KPMG (2018), los bancos españoles deberán implementar más de 100 regulaciones en el período 2018-2020. El sector financiero presentó también el 43\% de las operaciones unusuales o significativas debido a la gran concentración promovida igualmente por la regulación del sector (ej., adquisición del Banco Popular por Banco Santander, absorción de Banco Mare Nostrum S.A por Bankia, etc). Además, los sistemas de información han sido críticos en este sector. Así, esta tendencia ha sido seguida por la construcción en el IBEX-35 y en Tecnología, Medios y Telecomunicaciones en MC.

Tipos de riesgos. La tabla 8 muestra la amplia gama de riesgos reportados, desagregados entre las empresas del Ibex-35 y MC. Esto representa el número total de cada tipo de riesgo individual dentro de nuestra muestra.

TABLA 8

Análisis de los riesgos reportados en el informe ampliado de cotizadas españolas en 2017

\begin{tabular}{|c|c|c|c|c|}
\hline \multirow[b]{2}{*}{ Bloque B: KAM contable } & \multirow{2}{*}{ IBEX-35 } & \multirow{2}{*}{$\mathrm{MC}$} & \multicolumn{2}{|c|}{ Total } \\
\hline & & & Número & Porcentaje \\
\hline Fondo de comercio & 17 & 33 & 50 & $13 \%$ \\
\hline Activos intangibles & & 13 & 13 & $3 \%$ \\
\hline Inmov. material e inversiones inmobiliarias & 16 & 26 & 42 & $11 \%$ \\
\hline Inversiones en empresas del grupo y asociadas & 2 & 2 & 4 & $1 \%$ \\
\hline Inversiones financieras & 9 & 9 & 18 & $5 \%$ \\
\hline Activos por impuesto diferido & 11 & 33 & 44 & $12 \%$ \\
\hline Existencias & 1 & 15 & 16 & $4 \%$ \\
\hline Otros activos corrientes & & 3 & 3 & $1 \%$ \\
\hline Provisiones y pasivos contingentes & 19 & 19 & 38 & $10 \%$ \\
\hline Impuestos & 5 & 3 & 8 & $2 \%$ \\
\hline Deudas con entidades de crédito & & 4 & 4 & $1 \%$ \\
\hline Otros pasivos financieros & 3 & 8 & 11 & $3 \%$ \\
\hline Pensiones & 3 & & 3 & $1 \%$ \\
\hline Periodificaciones & & & & $0 \%$ \\
\hline Reconocimiento de ingresos & 16 & 42 & 58 & $16 \%$ \\
\hline Operaciones con partes vinculadas & & 1 & 1 & $0 \%$ \\
\hline Combinaciones de negocio & 8 & 12 & 20 & $5 \%$ \\
\hline Subtotal & 110 & 223 & 333 & $89 \%$ \\
\hline \multicolumn{5}{|l|}{ Bloque C: KAM General } \\
\hline Sistemas de información & 8 & 3 & 11 & $3 \%$ \\
\hline Cumplimiento legal y nomativo & 4 & 3 & 7 & $2 \%$ \\
\hline Cumplimiento condiciones contractuales & & 3 & 3 & $1 \%$ \\
\hline Transacciones inusuales o significativas & 4 & 3 & 7 & $2 \%$ \\
\hline Empresa en funcionamiento & & 4 & 4 & $1 \%$ \\
\hline Otros & 3 & 5 & 8 & $2 \%$ \\
\hline Subtotal & 19 & 21 & 40 & $11 \%$ \\
\hline Total & 129 & 244 & 373 & $100 \%$ \\
\hline
\end{tabular}

Fuente: elaboración propia. 
Las grandes empresas en España informan en su mayoría de dos a cuatro KAM y la mayoría de ellas incluyen el reconocimiento de ingresos, el deterioro del fondo de comercio y la recuperación de impuestos diferidos. Puesto que a menudo se trata de áreas críticas de los "juicios de la dirección", y de especial importancia para la valoración de las empresas, esto quizás no sea sorprendente. Además, son riesgos que afectan a todos los sectores. Estos tres riesgos representan el $41 \%$ del número total de riesgos (373) reportados por los auditores en este estudio.

Descripción del nivel de detalle en la descripción del riesgo. La NIA 700 exige que "para ser útiles a los usuarios de los estados financieros, las explicaciones de las KAM se describan... de manera que puedan relacionarse directamente con las circunstancias específicas de la entidad auditada y no sean, por tanto, cuestiones genéricas o abstractas expresadas en un lenguaje estandarizado". Se evaluaron las descripciones de las KAM en cada uno de los 131 informes de auditoría de este estudio. Este análisis muestra que existen diferencias entre las firmas de auditoría; por ejemplo, Deloitte parecía haber proporcionado descripciones de riesgo no estandarizadas más específicas, alrededor del 49\% de los riesgos detallados que citaron circunstancias específicas. Si se excluyera a KPMG del análisis se obtendría un desglose aproximado de 40/60 entre descripciones de riesgo específicas y genéricas. Esto podría ser un indicador de que hay margen de mejora en cuanto a la personalización de las descripciones de riesgos. En otras palabras, las descripciones de las KAM en las empresas españolas cotizadas fueron muy generales y similares (alrededor del $65 \%$ del total) de un informe de auditoría a otro, lo que disminuye la utilidad de la divulgación del riesgo.

Precisión. Algunos investigadores han analizado las características específicas del informe de auditoría como la longitud, legibilidad y consistencia lingǘstica. Los resultados empíricos mostraron que la incomprensibilidad del informe de auditoría generaban varios problemas que reducían la calidad informativa de los resultados de la auditoría (información asimétrica, falta de expectativas, ambigüedad...), así como la reputación profesional de los auditores (Fakhfakh, 2016b). Los inversores valoran especialmente la información cuando se presenta de forma concisa y en un lenguaje sencillo. Por lo tanto, se revisó el promedio de palabras para las descripciones de riesgo en cada informe: el número medio de palabras para las empresas del IBEX-35 es de 440 y 394 para las empresas de Mercado Continuo. Por término medio, las descripciones de riesgo tendían a ser más largas para las empresas del IBEX-35 que para las empresas de Mercado Continuo. Esto puede deberse a que se trata generalmente de empresas más grandes y complejas. En relación con las empresas de auditoría, PWC y EY tendían a incluir descripciones más largas de los riesgos en todos sus informes. Esto refleja la forma en que esas empresas de auditoría han tratado de proporcionar una mayor transparencia y detalle en la presentación de informes de riesgo, pero pone de relieve el reto de satisfacer las expectativas potencialmente contradictorias de los usuarios de los informes. Antes de la reforma de auditoría, los auditores no mostraban estas KAM porque formaban parte de información confidencial entre las empresas y las sociedades de auditoría incluidas en las cartas de recomendaciones. Actualmente, esta información está disponible para todos los lectores y también está estandarizada. Otra observación relevante es que las empresas de auditoría que no son Big-4 presentan descripciones más breves. Esto está en línea con las conclusiones de los estudios previos que demostraron que las Big-4 tienen mayores incentivos para emitir informes de auditoría más precisos en comparación con los pequeños auditores, reflejando una relación positiva entre el tamaño del auditor y la precisión del auditor (DeAngelo, 1981; Dye, 1993; Clive, 2012). 


\section{CONCLUSIONES E IMPLICACIONES PRÁCTICAS}

Analizar los riesgos de la información financiera a partir de las cuestiones clave de la auditoría identificadas por el auditor puede ser una buena herramienta para ayudar a los usuarios de la información financiera en su proceso de toma de decisiones. Por ello, este estudio se propuso analizar de forma práctica y sistemática el contenido del informe ampliado de auditoría con especial foco en las KAM descritas en el primer año de aplicación de la norma en España para las 131 empresas cotizadas. Según la muestra utilizada, se observó una tendencia generalizada a disponer de informes favorables, sin prácticamente párrafos de énfasis ni de empresa en funcionamiento, dado que el regulador (CNMV) ha presionado para que reflejen unos correctos estadosfinancieros debido a su gran repercusión económica en los mercados que cotizan. Esto está en línea con la dinámica actual tanto en España como en otros países incluidos Colombia, donde los requisitos para cotizar son cada vez más exigentes, debido principalmente a la última crisis económica mundial y a su impacto.

Una peculiaridad identificada en este estudio muestra que algunas firmas de auditoría describen la situación de empresa en funcionamiento como una KAM mientras que otras lo reflejan en un párrafo separado, lo que puede causar un distinto efecto en los usuarios de la información financiera porque si se describe como una KAM puede llamar menos la atención que si se sitúa en un párrafo separado. Las grandes empresas en España informan en su mayoría de dos a cuatro KAM y la mayoría incluye el reconocimiento de ingresos, el deterioro del fondo de comercio y la recuperación de impuestos diferidos. Esto es consistente con la importancia otorgada a las áreas de juicio de la dirección, y de particular importancia para la valoración de las empresas.

En cuanto al contenido, según la muestra analizada, existen grandes oportunidades de mejora en este ámbito al igual que ocurría en otros países como Holanda dada la generalidad de las descripciones y donde es posible que el regulador ponga foco en los próximos años. Atendiendo a los sectores, llama la atención en este estudio la relevancia del financiero, tanto en mayor número de KAM como en la longitud y especificidad de sus descripciones. Esto es debido a los mayores requisitos de la nueva regulación financiera así como las que entrarán en vigor en los próximos años, aproximadamente más de 100 regulaciones en el periodo 2018-2020.

Los resultados obtenidos tienen implicaciones interesantes tanto para otros países cuya implementación del informe ampliado de auditoría se producirá en el futuro como es el caso de Estados Unidos en el año 2019 o bien para aquellos países de implementación reciente que aún no han analizado en profundidad este nuevo informe, como es el caso de Colombia, según el análisis realizado a través de la base de datos Web of Science de Thomson Reuters $^{2}$. Este estudio permitirá comparar, entre otros, tanto el número de KAM descritos por el auditor así como la naturaleza y el nivel de detalle de los mismos. La utilidad de este informe puede extenderse tanto a los reguladores como a los analistas, auditores, gerentes e inversores dado que la información de los KAM es relevante tanto para tomar mejores decisiones en los negocios como para mejorar la relevancia de la auditoría.

Sin embargo, este trabajo no está exento de limitaciones. La muestra se refiere solamente a las empresas cotizadas en España, donde la mayoría son empresas matrices de filiales en el extranjero, en muchos casos, situadas en Latinoamérica. Un aspecto interesante sería analizar si existen diferencias en la aplicación de la nueva norma sobre informes de auditoría entre firmas de auditoría globales y locales. En general, las firmas de auditoría globales -Big4- suelen aplicar la misma metodología en todos los países para mantener la homogeneidad en sus informes. Este aspecto no ha sido analizado en este estudio y podría ser una línea de futura investigación.

\section{REFERENCIAS}

Asare, S. \& Wright, A. (2012). Investors', auditors', and lenders' understanding of the message conveyed by the standard audit report on the financial statements. Accounting Horizons, 26(2), 25-44. https://doi.org/10.2308/acch-50 138. 
Boolaky, P. \& Quick, R. (2016). Bank Directors' Perceptions of Expanded Auditor’s Reports. International Journal of Auditing, 20(1), 158-174. https://doi.org/10.1111/ijau.12063

Brasel, K., Doxey, M., Grenier, J. \& Reffett, A. (2016). Risk disclosure preceding negative outcomes: The effects of reporting critical audit matters on judgments of auditor liability. Accounting Review, 91(5), 1345-1362. https:/ /doi.org/10.2139/ssrn.2487396

Butler, M., Leone, A. \& Willenborg, M. (2004). An empirical analysis of auditor reporting and its association with abnormal accruals. Journal of Accounting and Economics, 37(2), 139-135. https://doi.org/10.2139/ssrn.298700.

Carson, E., Fargher, N., Geiger, M., Lennox, C., Raghunandan, K \& Willekens, M. (2013). Audit reporting for goingconcern uncertainty: A research synthesis. Auditing-A Journal of Practice and Theory, 32(1), 353-384. https:// doi.org/10.2308/ajpt-50324.

Christensen, B., Glover, S. \& Wood, D. (2012). Extreme estimation uncertainty in fair value estimates: Implications for audit assurance. Auditing-A Journal of Practice and Theory, 31(1), 127-146. https://doi.org/10.2308/ajpt-1 0191.

Clive L. (2012) Are large auditors more accurate than small auditors?, Accounting and Business Research, 29(3), 217-227. https://doi.org/10.1080/00014788.1999.9729582.

Clive S., Schmidt, J. \& Thompson, A. (2019). Are the expanded audit reports informative to investors? Evidence from the UK. Journal of Accounting and Economics, 3-53 (forthcoming). https://doi.org/10.2139/ssrn.2619785.

Coram, P., Mock, T., Turner, J. \& Gray, G. (2011). The Communicative Value of the Auditor's Report. Australian Accounting Review, 21(3), 235-252. https://doi.org/10.1111/j.1835-2561.2011.00140.x.

Cox, J. (2013). Strengthening financial reporting: An Essay on expanding the auditor's opinion letter. George Washington Law Review, 81(3), 1036-1062. Available at: https://scholarship.law.duke.edu/faculty_scholarshi $\mathrm{p} / 2869$

Czerney, K., Schmidt, J. \& Thompson, A. (2014). Does Auditor Explanatory Language in Unqualified Audit Reports Indicate Increased Financial Misstatement Risk? The Accounting Review, 89(6), 2115-2149. https://doi.org/10 $.2308 /$ accr-50836.

DeAngelo, L. (1981). Auditor size and audit quality. Journal of Accounting and Economics, 3(3), 183-199. https://do i.org/10.1016/0165-4101(81)90002-1.

Deumes, R., Schelleman, C., Vander B. \& Vanstraelen, A. (2012). Audit Firm Governance: Do Transparency Reports Reveal Audit Quality? Auditing: A Journal of Practice \& Theory, 31(4), 193-214. doi: 10.2308/ajpt-10301

Dye, R. (1993). Discussion: Limiting Auditors' Liability. Journal of Economics \& Management Strategy, 2, 435-443. h ttps://doi.org/10.1111/j.1430-9134.1993.00435.x.

Fakhfakh, M. (2016a). Linguistic features and legibility of the consolidated audit reports: An original investigation from the Tunisian case. Cogent Business and Management, 3(1), 655-687. https://doi.org/10.1080/23311975. 2016.1234360 .

Fakhfakh, M. (2016b). Understandability of unmodified audit report on consolidated financial statements: A normative and advanced study of the international normalization. Cogent Business and Management, 3, 1-25. $\mathrm{h}$ ttps://doi.org/10.1080/23311975.2016.1258134

FRC - Consejo de Información Financiera (2015). Extended auditor's reports. A review of experience in the first year.

FRC - Consejo de Información Financiera (2016). Extended auditor's reports. A further review of experience.

Garza Sánchez, H., Cortez, A., Méndez Sáenz, A \& Rodriguez García, M. (2016). Efecto en la calidad de la información ante cambios en la normatividad contable: caso aplicado al sector real mexicano. Contaduría y Administración, 62(3), 746-760. Doi: https://doi.org/10.1016/j.cya.2015.11.012.

Gray, G., Turner, J., Coram, P. \& Mock, T. (2011). Perceptions and misperceptions regarding the unqualified Auditor's Report by Financial Statement Preparers, users, and auditors. Accounting Horizons, 25(4), 659-684. https://do i.org/10.2308/acch-50060. 
Guiral-Contreras, A., Gonzalo-Angulo, J.A \& Rodgers, W. (2007). Information content and recently effect of the audit report in loan rating decisions. Accounting and Finance, 47(2), 285-304. https://doi.org/10.1111/j.1467-629x .2006.00208.x.

Gutierrez, E., Minutti-Meza, M., Tatum, K. \& Vulcheva, M. (2017). Consequences of adopting an expanded auditor's report in the United Kingdom. Review of Accounting Studies, Springer, 23(4), 1543-1587. https://doi.org/10.1 007/s11142-018-9464-0.

Hategan, C., Ioana, J., Renata Dana, N., Nicoleta, S. \& Iacobuta, A. (2015). The relevance of communication through independent auditor's report to the European business environment. Comparative study of Romania and Spain. Transformations in Business and Economics, 14 (2A), 378-392.

IAASB (2016). More Informative Auditor's Reports - What Audit Committees and Finance Executives Need to Know

IAASB (2015). The new auditor's report: enhancing auditor communications. Greater Transparency into the Financial Statement Audit. Available at: https://www.ifac.org/system/files/uploads/IAASB/Auditor-Reporting-Fact-Sh eet.pdf

ICAEW Audit and Assurance Faculty (2007). Audit Quality Fundamentals-Auditor reporting. Available at: https://www.icaew.com/-/media/corporate/files/technical/audit-and-assurance/audit-quality/audit-qualit $\mathrm{y}$-forum/january-2007-auditor-reporting.ashx

IFAC (2013). Reporting on Audited Financial Statements: Proposed New and Revised International Standards on Auditing. Exposure Draft. Available at: https://www.ifac.org/system/files/publications/files/All\%20Proposed \%20ISAs\%20Included\%20in\%20ED.pdf

Kachelmeier, S., Rimkus, D., Schmidt, J. \& Valentine, K. (2019). The Forewarning Effect of Critical Audit Matter Disclosures Involving Measurement. http://dx.doi.org/10.2139/ssrn.2481284

Kilgore, A., Barghathi, Y., Collison, D. \& Crawford, L. (2014). Earnings Management and the Role of the External Auditor. Conference: Financial Reporting and Business Communication FRBC At: Bristol, UK.

KPMG (2016). Nuevo informe de auditoria: mayor transparencia e información más relevante. Available at: https://as sets.kpmg.com/content/dam/kpmg/pdf/2016/03/nuevo-informe-auditoria.pdf

KPMG (2018). Claves de la regulación financiera: impacto y horizonte para las entidades de crédito. Julio 2018[Keys to financial regulation: impact and horizon for credit institutions. July 2018]. Available at: https://assets.kpmg .com/content/dam/kpmg/es/pdf/2018/07/claves-de-regulacion-financiera.pdf

Lennox, C., Wu, X. \& Zhang, T. (2015). The effect of audit adjustments on earnings quality: Evidence from China. Journal of Accounting and Economics, 61(2-3), 545-562. https://doi.org/10.1016/j.jacceco.2015.08.003.

Mock, T., Gray, G., Coram, P \& Turner, J. (2009). Perceptions and Misperceptions Regarding the Unqualified Auditor's Report by Financial Statement Preparers, Users, and Auditors. Accounting Horizons, 25(4), 659-684. https://d oi.org/10.2308/acch-50060

Muñoz-Izquierdo, N., Camacho-Miñano, M. \& Pascual-Ezama, D. (2017). The Content of the Audit Report in the Year Prior to Bankruptcy Filing. Empirical Evidence from Spain. Spanish Journal of Finance and Accounting, 46(1), 92-126. Available at SSRN: https://ssrn.com/abstract=2858851

PCAOB (2013). Release No. 2013-005 August 13, 2013 PCAOB Rulemaking Docket Matter No. 034. Available at: h ttps://pcaobus.org/Rulemaking/Docket034/Release_2013-005_ARM.pdf

PCAOB (2017). Release No. 2017-001 June 1, 2017 PCAOB Rulemaking Docket Matter No. 034. Available at: http s://pcaobus.org/Rulemaking/Docket034/2017-001-auditors-report-final-rule.pdf

Prasad, P. \& Chand, P. (2017). The Changing Face of the Auditor's Report: Implications for Suppliers and Users of Financial Statements. Australian Accounting Review, 27(4), 348-367. https://doi.org/10.1111/auar.12137.

Reid, L., Carcello, J., Chan, L. \& Neal, T. (2018). Impact of Auditor Report Changes on Financial Reporting Quality and Audit Costs: Evidence from the United Kingdom. Working paper SSNR. https://doi.org/10.2139/ssrn.2647507.

Ruiz-Barbadillo, E., Gómez-Aguilar, N. \& Aguilar-Contreras, G. (2002). Una comparación intersectorial y por tamaño de la calidad de la información contable a través del análisis del informe de auditoría [A cross-sector comparison and size comparison of the quality of accounting information through the analysis of the audit report.]. Revista Española de Financiación y Contabilidad, 31(112), 545-581. 
Sierra-Garci\#a, L., Gambetta, N., Garci\#a-Benau, M., \& Orta-Pe\#rez, M. (2019). Understanding the determinants of the magnitude of entity-level risk and account-level risk key audit matters: The case of the United Kingdom. The British Accounting Review, 51(3), 227-240. https://doi.org/10.1016/j.bar.2019.02.004.

Sikka, P. (2009). Financial crisis and the silence of the auditors. Accounting, Organizations and Society, 34(6-7), 868-873. https://doi.org/10.1016/j.aos.2009.01.004

Sirois, L., Bédard, J. \& Palash, B. (2017). The Informational Value of Key Audit Matters in the Auditor's Report: Evidence from an Eye-tracking Study. Accounting Horizons, 32(2), 141-162. https://doi.org/10.2308/acch-52 047

Zenzerovic, R. \& Valic-Vale, M. (2016). Diagnosing companies in financial difficulty based on the auditor's report. Croatian Operational Research Review, 7(1), 147-158. https://doi.org/10.17535/crorr.2016.0010.

\section{Notas}

* Artículo de investigación científica y tecnológica

1 La CNAE es una codificación obligatoria para todas las empresas presentes en el mercado europeo para poder hacer seguimiento de sus datos a fin de realizar encuestas, informes estadísticos y análisis públicos. Fue aprobada por el Real Decreto 475 del 13 de abril de 2007, por el que se aprueba la Clasificación Nacional de Actividades Económicas 2009.

2 La base de datos Web of Science de Thomson Reuters recoge las referencias de las principales publicaciones científicas de cualquier disciplina del conocimiento, tanto científico como tecnológico, humanístico y sociológicos desde 1945, esenciales para el apoyo a la investigación y para el reconocimiento de los esfuerzos y avances realizados por la comunidad científica y tecnológica.

\section{Licencia Creative Commons CC BY 4.0}

Para citar este artículo: Pérez-Pérez, Y., Camacho-Miñano, M. M. y Segovia-Vargas, M. J. (2019). Los nuevos informes ampliados de auditoría. Caso: las empresas cotizadas españolas. Cuadernos de Contabilidad, 20(49). https://doi.org/10.11144/Javeriana.cc20-49.ania 